\title{
Echocardiographic phenotype and prognosis in transthyretin cardiac amyloidosis
}

Liza Chacko ${ }^{a *}$ MBBS, Raffaele Martone ${ }^{b *}$ MD, Francesco Bandera ${ }^{c}$ MD PhD, Thirusha Lane $^{a} \mathrm{RN} \mathrm{PhD}$, Ana Martinez-Naharro ${ }^{\mathrm{a}} \mathrm{MD}$, Michele Boldrini ${ }^{\mathrm{a}} \mathrm{MD}$, Tamer Rezk ${ }^{\mathrm{a}} \mathrm{PhD}$, Carol Whelan ${ }^{\mathrm{a}} \mathrm{MD}$, Cristina Quarta ${ }^{\mathrm{a}} \mathrm{MD} \mathrm{PhD}$, Dorota Rowczenio ${ }^{\mathrm{a}} \mathrm{PhD}$, Janet A Gilbertson ${ }^{\mathrm{a}}$ CSci, Tanakal Wongwarawipat ${ }^{\mathrm{a}}$ MBBS, Helen Lachmann ${ }^{\mathrm{a}}$ MD, Ashutosh Wechelakar ${ }^{\mathrm{a}}$ MD, Sajitha Sachchithanantham ${ }^{\mathrm{a}}$ MD, Shameem Mahmood ${ }^{\mathrm{a}}$ MD, Rossella Marcucci MD PhD ${ }^{\mathrm{b}}$, Daniel Knight ${ }^{\mathrm{a}}$ MD, David Hutt ${ }^{\mathrm{a}}$ BAppS, James Moon ${ }^{\mathrm{d}}$ MD, Aviva Petrie ${ }^{\mathrm{e}} \mathrm{PhD}$, Francesco Cappelli ${ }^{\mathrm{b}}$ MD, Marco Guazzi ${ }^{\mathrm{c}}$ MD PhD, Philip N Hawkins ${ }^{\mathrm{a}}$ MD PhD, Julian D Gillmore ${ }^{\mathrm{a}}$ MD PhD, Marianna Fontana ${ }^{\mathrm{a} \bullet} \mathrm{MD} \mathrm{PhD}$

*Liza Chacko and Raffaele Martone contributed equally to this work.

-Julian Gillmore and Marianna Fontana contributed equally to this work.

${ }^{a}$ National Amyloidosis Centre, University College London, Royal Free Campus, London, UK ${ }^{\mathrm{b}}$ Tuscan Regional Amyloid Center, Careggi University Hospital (AOUC), Florence, Italy ${ }^{c}$ Cardiology University Department, Heart Failure Unit, IRCCS Policlinico San Donato, San Donato Milanese, Milan, Italy; Department for Biomedical Sciences for Health, University of Milano, Milan, Italy.

${ }^{\mathrm{d} B a r t s}$ Heart Centre, St Bartholomew's Hospital, West Smithfield, London, UK

eEastman Dental Institute, University College London, Grays Inn Road, London WC1X 8LD, UK

Disclosures: M Fontana is supported by a British Heart Foundation Intermediate Clinical Research Fellowship (FS/18/21/33447).

\section{Address for correspondence:}

Dr Marianna Fontana,

National Amyloidosis Centre, University College London, Royal Free Campus,

Rowland Hill Street, London, NW3 2PF, UK

E-mail: m.fontana@ucl.ac.uk

Phone No: +44 2074332764

Fax No: +44 2044332817 


\section{Abbreviations:}

ATTR-CM: transthyretin amyloidosis cardiomyopathy wtATTR: wild type transthyretin hATTR: hereditary transthyretin HFpEF: heart failure with preserved ejection fraction EMB: endomyocardial biopsy RWT: relative wall thickness

PWTd: posterior wall thickness in diastole MCF: myocardial contraction fraction

SV: stroke volume

LAA: left atrial area

RAA: right atrial area

EF: ejection fraction

MAPSE: mitral annular plane systolic excursion

TAPSE: tricuspid annular plane systolic excursion

PASP: pulmonary artery systolic pressure

TR: tricuspid regurgitation

LS: longitudinal strain

SAB: systolic apex to base

RALS: relative apical longitudinal strain

AVA: aortic valve area

MR: mitral regurgitation

IVSd: interventricular septum in diastole

TAVI: transcatheter aortic valve implantation 


\section{INTRODUCTION}

Transthyretin amyloidosis cardiomyopathy (ATTR-CM) is a progressive and ultimately fatal cardiomyopathy characterised by deposition in the myocardial extracellular space of amyloid fibrils derived from plasma transthyretin. ATTR-CM can be classified as non-hereditary, associated with the deposition of wild-type transthyretin (wtATTR) or hereditary, associated with genetic variants of TTR (hATTR). ${ }^{1,2}$ Historically there has been a striking mismatch between the frequency with which the diagnosis of ATTR-CM has been made during life compared with previous autopsy series in which cardiac ATTR amyloid deposits have been reported in up to $25 \%$ of elderly individuals. ${ }^{3}$ In recent years however, greater awareness of ATTR-CM as an under-recognised cause of heart failure (HF), coupled with advances in diagnostic techniques have resulted in a dramatic increase in diagnosis of ATTR-CM in patients presenting with heart failure with preserved ejection fraction (HFpEF). Estimates of prevalence range from $13 \%$ to $16 \%$ in cohorts of patients with $\mathrm{HFpEF}^{4}$ and severe AS requiring transcatheter aortic valve replacement respectively. ${ }^{5}$

ATTR-CM can now be diagnosed without need for histology, avoiding the risks associated with endomyocardial biopsy (EMB) which was previously required. ${ }^{6}$ The diagnosis of ATTR-CM calls upon multimodality imaging techniques: cardiac MRI and bone scintigraphy produce highly characteristic images and both play a major role in the diagnosis of suspected ATTR-CM. ${ }^{7,8}$ Whilst bone scintigraphy is widely available and inexpensive, the widespread use of CMR remains limited by lack of infrastructure and cost implications.Worldwide, echocardiography remains the most accessible first line investigative tool for patients with HF, including those with ATTR-CM among whom it has traditionally been associated with poor sensitivity and specificity. ${ }^{9}$ Despite this however, several echocardiographic features are reported to be prognostic, ranging from parameters of 
conventional left ventricular (LV) remodelling and of diastolic dysfunction to deformationbased parameters. ${ }^{10-15}$ These prognostic indices have been determined either in studies focusing on single variables or in small retrospective series leaving an important knowledge gap on the echocardiographic phenotype of ATTR-CM as a whole and the relative pathophysiological importance of different mechanisms in driving the clinical characteristics and prognosis of the disease.

The aims of the present study were: (1) To describe the functional and structural echocardiographic cardiac abnormalities at the time of diagnosis across a spectrum of wildtype and hereditary ATTR-CM; (2) To assess the structural and functional echocardiographic parameters independently associated with prognosis in a large cohort of patients with wildtype and hereditary ATTR-CM. 


\section{METHODS}

\section{Patient Population}

Patients referred to the National Amyloidosis Centre (NAC), Royal Free Hospital, London, United Kingdom between 2000 and 2019 in whom ATTR-CM was confirmed on the basis of validated diagnostic criteria ${ }^{6}$ were invited to participate in a prospective protocolised clinical follow up program comprising systematic evaluation of cardiac parameters and survival. Briefly, the diagnosis of ATTR-CM was established on the basis of presence of symptoms of heart failure together with a characteristic amyloid echocardiogram and either direct EMB proof of ATTR amyloid, or presence of ATTR amyloid in an extra-cardiac biopsy along with cardiac uptake on 99mTechnetium labelled 3,3-diphosphono-1,2propanodicarboxylic acid(99mTc-DPD) scintigraphy, or Perugini grade 2 or 3 cardiac uptake on 99mTc-DPD scintigraphy in the absence of an abnormal serum free light chain ratio and monoclonal immunoglobulin in the serum and urine by immunofixation. ${ }^{6}$ All patients who were diagnosed with ATTR-CM underwent sequencing of the TTR gene at the time of diagnosis as previously described. ${ }^{1}$ Patients who did not consent to the protocolised clinical follow up program at NAC and those who received disease modifying therapy including orthotopic liver transplantation for hATTR amyloidosis, TTR stabilizer therapy for more than 6 months, or a TTR-lowering therapy (within the context of a clinical trial) were excluded from the study.

Patients were managed in accordance with the Declaration of Helsinki and provided written informed consent for retrospective analysis and publication of their data with approval from the Royal Free Hospital ethics committee (ref: 06/Q0501/42). 
Echocardiography. Echocardiographic evaluation was performed using a GE Vivid E9 ultrasound machine equipped with a $5 \mathrm{~S}$ probe and measurements performed offline using EchoPAC software (Version 202). At least 3 consecutive beats were recorded for each view, and images were stored for off-line analysis. All echocardiograms were reviewed by experienced operators blinded to the final diagnosis and analysed according to guidelines (details for non-deformation parameters provided in the supplemental material).

Digitally acquired clips were considered suitable for offline 2D Speckle Strain Imaging analysis if at least three cardiac cycles were available, with high frame rates (70 to 100 frame/s) and without dropout of more than one LV segment or significant foreshortening of the ventricle. The endocardial border was traced at the end-diastolic frame in the apical view. End-diastole was defined by the QRS complex or by the frame just before mitral valve closure. The software tracked speckles along the endocardial and epicardial borders throughout the cardiac cycle, and the width of the region of interest was adjusted to fit the entire myocardium. All strain and strain-derived variables were measured in the apical 4chamber view. Peak longitudinal strain (LS) was computed automatically, generating regional data from 6 segments (basal, mid, apical interventricular septum and basal, mid, apical lateral wall), to calculate an average value. Strain-derived variables were acquired and calculated according to previous studies: septal longitudinal systolic apex to base (SAB) ratio ${ }^{16}$ and relative apical longitudinal strain (RALS) as the average 4-chamber apical segments peak longitudinal strain/average basal and mid 4-chamber peak longitudinal strain.${ }^{17}$ Valvular assessment was performed using an integrated approach as per current guidelines. 


\section{Statistical analysis}

All mortality data were obtained via the UK Office of National Statistics. The mortality endpoint was defined as time to death from baseline for all deceased patients and time to Censor date, $24^{\text {th }}$ of April 2019, from baseline among the remainder. Follow up was restricted to $\leq 60$ months, after which patients were censored due to the low number of patients at risk after 60 months. The three genotypic sub-groups of interest were wild-type ATTR cardiomyopathy (wtATTR-CM), V122I-associated hereditary ATTR cardiomyopathy (V122I-hATTR-CM) and T60A-associated hereditary ATTR cardiomyopathy(T60AhATTR-CM).

As a number of the numerical variables had skewed distributions, a Kruskal Wallis test was used to compare the distributions of each of the numerical variables at baseline in the three genotypic subgroups. A significant result was followed by Bonferroni corrected Mann Whitney pairwise comparisons to establish where the differences lay.

Survival was evaluated with Cox proportional hazards regression analysis, providing estimated hazard ratios with 95\% confidence intervals (CIs) and Kaplan-Meier curves. Fifteen echocardiographic variables were selected a priori based upon clinical relevance: SV index, EF, MCF, LAA index, RAA index, interventricular septum in diastole (IVSd), significant TR, significant MR, LS, E/e', TAPSE, PASP, TAPSE/PASP, severe aortic stenosis (AS) and regional wall thickness (RWT). The proportional hazards assumption was checked and confirmed. The echocardiographic variables were first explored with univariate Cox regression analysis. The variables that were statistically significant predictors of outcome on simple Cox regression analysis were entered into a multivariable Cox proportional hazards analysis to determine which covariates were independent predictors of mortality. Possible collinearity among candidate predictors was assessed using variance inflation factors with threshold equal to 5. In order to avoid statistical coupling of variables, 
separate multivariable models were performed that excluded parameters derived from one another (for example, indexed SV, LVEF and MCF). Harrell's statistic was calculated for the different models.

The multivariable Cox proportional hazards analysis with the selected variables was initially performed in the overall population and was then repeated in the population in which patients with severe AS were omitted and in the two genotypic sub-groups wtATTR-CM and V122I-hATTR-CM. The analysis was not applied to T60A-hATTR-CM patients due to the low number of patients and events.

All data were analysed using Stata software (StataCorp. 2017. Stata Statistical Software: Release 15. College Station, TX: StataCorp LLC). A significance level of 0.05 was used for all hypothesis tests unless otherwise stated. 


\section{Results}

\section{Characteristics of the cohort at baseline and comparison of genotypes}

There were 1240 patients in the whole study cohort, 766(62\%) with wild-type ATTR-CM (wtATTR-CM) (age 77.5(SD 6.7), male 94\%), 314(25\%) with V122I-associated hATTR-CM (age 75.0(SD 7.4), male 73\%), 127(10\%) with T60A-associated hATTR-CM (age 66.0 (SD 7.8), male 68\%), 33(3\%) with non-V122I non T60A-associated hATTR-CM (age 58.64 (SD 11.93), male $82 \%$ ). The main reason for exclusion from the study was a refusal to consent to the protocolized program of follow up at the NAC. Thirty patients were excluded due to receipt of disease modifying therapy (liver transplantation, diflunisal, anti-sense oligonucleotide therapy or RNA inhibitor therapy). There was no difference in diseaserelated parameters or disease severity between patients with ATTR-CM who were included and excluded from the study. The study patients with non-V122I-non T60A-associated hATTR-CM had the following mutations: G47V, V30M, S77Y, I73V, A97S, A120S, D39V, E89K, H90D, I107V, F33I, S50R, V20I.

At diagnosis, patients with wtATTR-CM, V122I-associated and T60A-associated hATTR-CM differed significantly in terms of remodelling of the cardiac chambers, LV systolic and diastolic function, right ventricular (RV) function and pulmonary pressures (Table 1). Specifically, compared to wtATTR-CM patients, patients with V122I-hATTR-CM, in spite of similar degree of increase in the wall thickness (LV septum, LV posterior wall) showed a more severe dysfunction, across a wide range of non-deformation and deformation based parameters, of LV systolic function, diastolic function, RV function, pulmonary pressure, and mitral and tricuspid regurgitation. On the contrary, compared to wtATTR-CM patients, patients with T60A-hATTR-CM, in spite of a similar degree of increase in wall thickness, had significantly better LV systolic and diastolic function, RV systolic function, and milder degrees of bi-atrial dilatation and tricuspid regurgitation. 


\section{Survival from baseline}

At follow up (mean 32 \pm 18 months), 489(39\%) of 1240 patients had died, including 283 (37\%) in the wtATTR-CM group, 156(50\%) in the V122I-hATTR-CM group, 43(34\%) in the T60A-hATTR-CM, and 7(21\%) in the non-T60A non V122I group.

Median patient survival from diagnosis by Kaplan-Meier analysis was 58 months in patients with wtATTR-CM compared to 36 months among patients with V122I-hATTR-CM and >60 months in T60A-hATTR-CM. Survival probability in the overall population at 24 months was $0.7795 \%$ CI $0.75-0.80$ and at 36 months $0.6695 \%$ CI $0.62-0.68$. Survival probability in wtATTR-CM group at 24 months was $0.8195 \% \mathrm{CI} 0.78-0.84$ and at 36 months $0.7095 \% \mathrm{CI}$ 0.67-0.74. Survival probability in T60A-hATTR-CM group at 24 months was $0.7495 \%$ CI 0.69-0.85 and at 36 months $0.7095 \%$ CI 0.60-0.78. Survival probability in V122I-hATTRCM group at 24 months was $0.6695 \%$ CI $0.60-0.71$ and at 36 months 0.49 95\%CI 0.43-0.56.

Twelve significant parameters explored with univariate Cox regression analysis were used in the multivariable model (Table 2). In order to avoid statistical coupling of variables, separate multivariable models were performed that excluded coupled parameters (for example 3 separate models were created for SV index, LVEF and MCF, see supplemental material Table 1). For all variables variance inflation factor was less than 3. Amongst the three models with SV index, LVEF and MCF, the one with SV index was chosen for marginally better c-statistics. A multivariable model combining LAA index, RAA index, IVSd, significant TR, significant MR, LS, E/e', TAPSE/PASP, SV index, RWT, HR and severe AS at the time of diagnosis revealed that SV index (HR 0.97; 95\% CI 0.95-0.99, $\mathrm{p}=0.004)$, RAA index (HR 1.05; 95\% CI 1.01-1.10 p=0.016), LS (HR 1.08; 95\% CI 1.04 $1.12 \mathrm{p}<0.001)$, and severe AS (HR 2.46; 95\% CI, 1.29- 4.72, $\mathrm{p}=0.007$ ) were independently associated with patient survival in the overall population. (Table 3). LS, SV index and AS 
remained independently associated with patient survival in the overall populations also after adjustment for NYHA class and the validated staging system that uses eGFR and NTproBNP (Table 4). When patients with AS were removed from the analysis, E/e' (HR 1.02; 95\% CI, 1.00-1.03, $\mathrm{p}=0.033$ ) also became independently associated with patient survival, with no changes in the other predictors. (Table 3)

The same multivariable model was used in wtATTR-CM and V122I-hATTR-CM patients separately (Table 5). The model was not applied to non-V122I-hATTR-CM patients due to low numbers of events. In wtATTR-CM patients, RAA index, LS and E/e' were significantly associated with mortality (HR 1.07, 95\% CI 1.01-1.13, p=0.023; HR 1.07, 95\% CI 1.03-1.13, $\mathrm{p}=0.004 ;$ HR $1.03 ; 95 \%$ CI 1.00-1.05, $\mathrm{p}=0.019$, respectively) whilst in patients with V122IhATTR-CM, RAA index and LS remained independently associated with survival (HR 1.10, 95\% CI 1.02-1.19, p=0.015; HR 1.10, 95\% CI 1.03-1.18, p=0.004, respectively).

Eighty-five patients were diagnosed with aortic stenosis, 21 with mild AS, 42 with moderate AS and 22 with severe AS. Of the patients with severe AS, 11 had low flow-low gradient AS, 9 had paradoxically low flow AS and 2 patients had high gradient AS . Of the patients with severe AS at the time of diagnosis, 1 patient had surgical aortic valve replacement (survival 6 months) and 5 patients had transcatheter aortic valve implantation (TAVI). There was a statistically significant difference in survival between patients who underwent TAVI and patients who did not undergo any treatment for the severe AS $(\mathrm{p}=0.012)$. Figure 3. 


\section{Discussion}

This study of more than 1000 patients with ATTR-CM highlights the relative prognostic importance of a range of different echocardiographic parameters at the time of diagnosis and yields many new insights into the complexity of the disease pathophysiology which may, in part, explain its phenotypic heterogeneity.

The three most common UK (and US) genotypic subgroups included in the study are wtATTR-CM, V122I-hATTR-CM and T60A-hATTR-CM. Patients with V122I-hATTR-CM present with the most severe dysfunction across all echocardiographic parameters, T60AhATTR-CM patients have better cardiac function and those with wtATTR-CM present with intermediate dysfunction. Our results demonstrate that that these three subtypes present with varying degrees of severity which may reflect either differences in the time of presentation or intrinsic differences in disease biology between subtypes. However, a few observations from the present study are in favour of the latter for patients with V122I ATTR-CM. Firstly, although patients with V122I had similar increases in LV wall thickness as ATTRwt, these patients had significantly lower indices of LV function (stroke volume, ejection fraction, $\mathrm{MCF}$ ), worse diastolic function, increased incidence of moderate and severe mitral and tricuspid regurgitation, as well as increased pulmonary pressures, indicating more advanced cardiac disease overall. Secondly, the comparable atrial dimensions observed between patients with V122I ATTR-CM and wtATTR-CM despite almost two-fold higher prevalence of moderate to severe MR and TR suggests impaired compensatory atrial dilatation in response to valvular regurgitation which may be explained by atrial amyloid infiltration. Thirdly, in spite of similar increase in LV wall thickness, the left ventricular remodelling is different, with V122I ATTR-CM showing a more severe degree of concentric remodelling, with small LVEDD and increased RWT, compared to wtATTR-CM. These observations combined with the knowledge that patients with hATTR-CM are diagnosed more rapidly 
following the onset of cardiac symptoms than those with wtATTR-CM ${ }^{18}$ strongly supports the hypothesis that there exists an inherent difference in the disease biology. On the other hand, patients with T60A-hATTR-CM had better biventricular systolic function, less compromised diastolic dysfunction, a minor degree of TR and a minor degree of increase in pulmonary pressure with a similar degree of increase in the ventricular wall thickness. The less severe disease phenotype in T60A may be explained by differences in time to presentation, in which neurological symptoms rather than cardiac symptoms often lead to first presentation.

Cardiac ATTR amyloidosis has been commonly considered a form of restrictive cardiomyopathy and the pathophysiology of heart failure has traditionally been attributed to diastolic dysfunction. ${ }^{19}$ Few studies have assessed the individual contributions of systolic and diastolic dysfunction to the pathophysiology of ATTR-CM, including studies on the prognostic role of longitudinal strain ${ }^{20}$ (NEED more REF\#) but no study has comprehensively assessed the relative contribution of multiple echocardiographic parameters that reflect overall cardiac performance. Our findings suggest a complex pathophysiological model where both LV systolic and diastolic function are all independently associated with prognosis, with the systolic dysfunction representing a marker of advanced disease. These findings mimic the haemodynamic model associated with cardiac amyloidosis in which progressive extracellular amyloid infiltration alters myocardial stiffness, resulting in an upward and leftward shift in the end-diastolic pressure-volume relationship, with concomitant declines in stroke volume. More complex is the correlation between RV sided parameters and mortality: the absence of association between PASP and mortality is intriguing and possibly reflects the relatively high prevalence of advanced right ventricular dysfunction associated with severe TR. On the contrary, RA remains independently predictive of mortality, and this probably reflects the complex nature of this parameter, with 
RA dilatation being the final common result of different pathogenetic mechanisms such as RV dysfunction and dilatation, pressure/volume overload and RV-pulmonary circulation uncoupling. Importantly, LS, SV index and AS remained independently associated with patient survival in the overall populations also after adjustment for NYHA class and the validated staging system that uses eGFR and NT-proBNP, highlighting their independent prognostic role beyond current staging systems. Finally it is interesting to observe the relatively low Harrel C-statistics of models containing only echocardiographic variables. This is not surprising, as cardiac ATTR amyloidosis is a complex disease with multiorgan involvement, where a combination of factors beyond cardiac amyloid infiltration and related dysfunction, including autonomic dysfunction, activation of the neuro-hormonal axis and renal perfusion, all interact to predict patient prognosis.

Interestingly, when the multivariable model is applied to wtATTR-CM and V122I hATTR-CM, LS remained consistently associated with prognosis, supporting the role of myocardial deformation imaging as a sensitive tool for characterizing LV dysfunction in ATTR amyloidosis over more traditional echocardiographic parameters (Figure 1). ${ }^{9}$

This is the first study to demonstrate the independent prognostic role of severe aortic stenosis in patients with ATTR-CM. In patients with both AS and ATTR CM, there have been uncertainties as to which disease process was the primary driver of poor outcome. Along with a wider awareness of co-existent cardiac amyloidosis and aortic stenosis, this knowledge gaps translates into clinical uncertainty on the optimal management of patients in this group, specifically whether or not to consider aortic valve intervention in patients with ATTR-CM. Our study indicates that presence of severe AS in the context of ATTR-CM is associated with a dramatic reduction in patient survival (Figure 2) and suggests that the aortic stenosis drives the poor outcome in patients with both diseases (median survival 22 vs 53 months). Of interest, patients who underwent TAVI had significantly longer survival than 
patients that did not receive any intervention, but this is in very small patient number and potentially affected by selection biases. However, the independent prognostic role of severe AS encourages the need for further studies assessing the potential benefit of aortic valve replacement procedures in patients with both diseases.

In summary, ATTR-CM, traditionally considered a predominantly diastolic heart disease, is characterised by a significantly more complex pathophysiology, in which multiple processes within the myocardium are compromised with each being more or less prominent at different timepoints, depending upon the individual, their co-morbidities and the genotype. This advancement in our understanding of the disease pathophysiology is especially timely since several treatments for ATTR-CM which hold great promise in transforming the traditionally accepted model of ATTR CM as a restrictive cardiomyopathy are becoming available to patients. 


\section{Study limitations}

The stroke volume was measure based on estimates of LV volumes and thus does not account for valvular regurgitation, leading to overestimation of SV in the presence of significant MR. Although Doppler derived SV could have overcome this limitation, this was not available in the present population. However, several studies both within amyloidosis and with focus on MCF have used the LVEDV-LVESV formula, sometimes using the Teichholz methods to assess volumes which is a far less accurate method of deriving SV than the method that we used. $^{15,21,22}$ Finally, echocardiographic studies have suggested that in patients with aortic stenosis and increased basal interventricular septum thickness, LVOT geometry may be irregular and may not be efficaciously assessed by two dimensional echocardiography, leading to SV over estimation. The asymmetric septal increase in the wall thickness in ATTR amyloidosis could act similarly, eventually reducing the reliability of Doppler LVOT derived $\mathrm{SV} .{ }^{23}$ Furthermore, on the multivariate analysis we adjust for MR, and this at least in part should account for this problem.

Strain analysis was performed only in a 4-chamber view. This approach was preferred to the global LS to minimise excluded patients, as good quality imaging without segment drop out is more challenging in 2- or 3-chamber apical views compared to 4-chamber views. ${ }^{24}$ Additionally, a good correlation between GLS in the three apical views and 4-chamber longitudinal strain has been demonstrated.$^{24}$ Strain analysis was performed using a single vendor software (Echo PAC software, GE), and whilst we acknowledge that intervendor variability has been reported, it has been demonstrated that mean LV global LS values obtained from the software used in this study are not significantly different from other mainstream providers. ${ }^{25} \mathrm{SV}$ was calculated as: EDV-ESV. This is associated with an overestimation of the SV when MR is present. 
Conflict of Interest: none declared 


\section{REFERENCES}

1. Rowczenio DM, Noor I, Gillmore JD, Lachmann HJ, Whelan C, Hawkins PN, Obici L, Westermark P, Grateau G, Wechalekar AD. Online registry for mutations in hereditary amyloidosis including nomenclature recommendations. Hum Mutat 2014;35:E2403-12.

2. Ruberg FL, Grogan M, Hanna M, Kelly JW, Maurer MS. Transthyretin Amyloid Cardiomyopathy: JACC State-of-the-Art Review. J Am Coll Cardiol 2019;73:2872-2891.

3. Cornwell GG, 3rd, Murdoch WL, Kyle RA, Westermark P, Pitkanen P. Frequency and distribution of senile cardiovascular amyloid. A clinicopathologic correlation. Am J Med $1983 ; 75: 618-23$.

4. Gonzalez-Lopez E, Gallego-Delgado M, Guzzo-Merello G, de Haro-Del Moral FJ, CoboMarcos M, Robles C, Bornstein B, Salas C, Lara-Pezzi E, Alonso-Pulpon L, Garcia-Pavia P. Wild-type transthyretin amyloidosis as a cause of heart failure with preserved ejection fraction. Eur Heart J 2015;36:2585-94.

5. Castaño A ND, Hamid N, Khalique OK, Morgenstern R, DeLuca A, Rubin J, Chiuzan C, Nazif T, Vahl T, George I, Kodali S, Leon MB, Hahn R, Bokhari S, Maurer MS. Unveiling transthyretin cardiac amyloidosis and its predictors among elderly patients with severe aortic stenosis undergoing transcatheter aortic valve replacement. . Eur Heart J 2017;38:2879-87.

6. Gillmore JD, Maurer MS, Falk RH, Merlini G, Damy T, Dispenzieri A, Wechalekar AD, Berk JL, Quarta CC, Grogan M, Lachmann HJ, Bokhari S, Castano A, Dorbala S, Johnson GB, Glaudemans AW, Rezk T, Fontana M, Palladini G, Milani P, Guidalotti PL, Flatman K, Lane T, Vonberg FW, Whelan CJ, Moon JC, Ruberg FL, Miller EJ, Hutt DF, Hazenberg BP, Rapezzi C, 
Hawkins PN. Nonbiopsy Diagnosis of Cardiac Transthyretin Amyloidosis. Circulation 2016;133:2404-12.

7. Maurer MS. Noninvasive Identification of ATTRwt Cardiac Amyloid: The Reemergence of Nuclear Cardiology. Am J Med 2015;128:1275-80.

8. Martinez-Naharro A, Treibel TA, Abdel-Gadir A, Bulluck H, Zumbo G, Knight DS, Kotecha T, Francis R, Hutt DF, Rezk T, Rosmini S, Quarta CC, Whelan CJ, Kellman P, Gillmore JD, Moon JC, Hawkins PN, Fontana M. Magnetic Resonance in Transthyretin Cardiac Amyloidosis. J Am Coll Cardiol 2017;70:466-477.

9. Pagourelias ED, Mirea O, Duchenne J, Van Cleemput J, Delforge M, Bogaert J, Kuznetsova T, Voigt JU. Echo Parameters for Differential Diagnosis in Cardiac Amyloidosis: A Head-to-Head Comparison of Deformation and Nondeformation Parameters. Circ Cardiovasc Imaging 2017;10:e005588.

10. Tendler A, Helmke S, Teruya S, Alvarez J, Maurer MS. The myocardial contraction fraction is superior to ejection fraction in predicting survival in patients with AL cardiac amyloidosis. Amyloid 2015;22:61-6.

11. Siepen FAD, Bauer R, Voss A, Hein S, Aurich M, Riffel J, Mereles D, Rocken C, Buss SJ, Katus HA, Kristen AV. Predictors of survival stratification in patients with wild-type cardiac amyloidosis. Clin Res Cardiol 2018;107:158-169.

12. Ruberg FL, Maurer MS, Judge DP, Zeldenrust S, Skinner M, Kim AY, Falk RH, Cheung KN, Patel AR, Pano A, Packman J, Grogan DR. Prospective evaluation of the morbidity and mortality of wild-type and V122I mutant transthyretin amyloid cardiomyopathy: the Transthyretin Amyloidosis Cardiac Study (TRACS). Am Heart J 2012;164:222-228 e1.

13. Knight DS, Zumbo G, Barcella W, Steeden JA, Muthurangu V, Martinez-Naharro A, Treibel TA, Abdel-Gadir A, Bulluck H, Kotecha T, Francis R, Rezk T, Quarta CC, Whelan CJ, 
Lachmann HJ, Wechalekar AD, Gillmore JD, Moon JC, Hawkins PN, Fontana M. Cardiac

Structural and Functional Consequences of Amyloid Deposition by Cardiac Magnetic Resonance and Echocardiography and Their Prognostic Roles. JACC Cardiovasc Imaging 2019;12:823-833.

14. Binder C, Duca F, Stelzer PD, Nitsche C, Rettl R, Aschauer S, Kammerlander AA, Binder T, Agis H, Kain R, Hengstenberg C, Mascherbauer J, Bonderman D. Mechanisms of heart failure in transthyretin vs. light chain amyloidosis. Eur Heart J Cardiovasc Imaging 2019;20:512-524.

15. Rubin J, Steidley DE, Carlsson M, Ong ML, Maurer MS. Myocardial Contraction Fraction by M-Mode Echocardiography Is Superior to Ejection Fraction in Predicting Mortality in Transthyretin Amyloidosis. J Card Fail 2018;24:504-511.

16. Liu D, Hu K, Niemann M, Herrmann S, Cikes M, Stork S, Gaudron PD, Knop S, Ertl G, Bijnens B, Weidemann F. Effect of combined systolic and diastolic functional parameter assessment for differentiation of cardiac amyloidosis from other causes of concentric left ventricular hypertrophy. Circ Cardiovasc Imaging 2013;6:1066-72.

17. Phelan D, Collier P, Thavendiranathan P, Popovic ZB, Hanna M, Plana JC, Marwick TH, Thomas JD. Relative apical sparing of longitudinal strain using two-dimensional speckletracking echocardiography is both sensitive and specific for the diagnosis of cardiac amyloidosis. Heart 2012;98:1442-8.

18. Lane T, Fontana M, Martinez-Naharro A, Quarta CC, Whelan CJ, Petrie A, Rowczenio DM, Gilbertson JA, Hutt DF, Rezk T, Strehina SG, Caringal-Galima J, Manwani R, Sharpley FA, Wechalekar AD, Lachmann HJ, Mahmood S, Sachchithanantham S, Drage EPS, Jenner HD, McDonald R, Bertolli O, Calleja A, Hawkins PN, Gillmore JD. Natural History, Quality of Life, and Outcome in Cardiac Transthyretin Amyloidosis. Circulation 2019;140:16-26. 
19. Gertz MA, Benson MD, Dyck PJ, Grogan M, Coelho T, Cruz M, Berk JL, Plante-

Bordeneuve V, Schmidt HHJ, Merlini G. Diagnosis, Prognosis, and Therapy of Transthyretin Amyloidosis. J Am Coll Cardiol 2015;66:2451-2466.

20. Quarta CC, Solomon SD, Uraizee I, Kruger J, Longhi S, Ferlito M, Gagliardi C, Milandri A, Rapezzi C, Falk RH. Left ventricular structure and function in transthyretin-related versus light-chain cardiac amyloidosis. Circulation 2014;129:1840-9.

21. King DL, El-Khoury Coffin L, Maurer MS. Myocardial contraction fraction: a volumetric index of myocardial shortening by freehand three-dimensional echocardiography. J Am Coll Cardiol 2002;40:325-9.

22. Shimada YJ, Hoeger CW, Latif F, Takayama H, Ginns J, Maurer MS. Myocardial Contraction Fraction Predicts Cardiovascular Events in Patients With Hypertrophic Cardiomyopathy and Normal Ejection Fraction. J Card Fail 2019;25:450-456.

23. Koto D, Izumo M, Machida T, Suzuki K, Yoneyama K, Suzuki T, Kamijima R, Kobayashi Y, Harada T, Akashi YJ. Geometry of the left ventricular outflow tract assessed by 3D TEE in patients with aortic stenosis: impact of upper septal hypertrophy on measurements of Doppler-derived left ventricular stroke volume. J Echocardiogr 2018;16:162-172.

24. Salaun E, Casalta AC, Donal E, Bohbot Y, Galli E, Tribouilloy C, Hubert S, Magne J, Mancini J, Renard S, Avierinos JF, Maysou LA, Lavoute C, Szymanski C, Haentjens J, Habib G. Apical four-chamber longitudinal left ventricular strain in patients with aortic stenosis and preserved left ventricular ejection fraction: analysis related with flow/gradient pattern and association with outcome. Eur Heart J Cardiovasc Imaging 2018;19:868-878.

25. Shiino K, Yamada A, Ischenko M, Khandheria BK, Hudaverdi M, Speranza V, Harten M, Benjamin A, Hamilton-Craig CR, Platts DG, Burstow DJ, Scalia GM, Chan J. Intervendor 
consistency and reproducibility of left ventricular 2D global and regional strain with two different high-end ultrasound systems. Eur Heart J Cardiovasc Imaging 2017;18:707-716. 


\section{Figure Legends}

Figure 1. Kaplan-Meier curves displaying event-free survival in the overall population according to Longitudinal Strain $\geq-10 \%$ or $<-10 \%$

Figure 2. Prognostic impact of Aortic Stenosis in Cardiac Amyloidosis. Kaplan-Meier curves displaying event-free survival in the overall population according to presence or absence of severe aortic stenosis

Figure 3. Prognostic impact of TAVI on survival in patients with severe aortic stenosis in Cardiac Amyloidosis. Kaplan-Meier curves displaying event-free survival according to presence or absence of a TAVI procedure

\section{Text Tables}

Table 1. Echocardiographic Findings in Patients With Wild type, T60A and V122I Cardiac Amyloidosis

Table 2. Univariable Cox Regression Analysis of Risk of Death in the Overall Population.

Table 3. Multivariable Cox Regression Analysis of Risk of Death in the Overall Population, According to Presence or Absence of Severe Aortic Stenosis.

Table 4. Multivariable Cox Regression Analysis of Risk of Death in the Overall Population adjusting for NYHA and NAC stage (SV Model).

Table 5.Multivariable Cox Regression Analysis of Risk of Death in Wild type and V122I subgroups. 
Figure 1

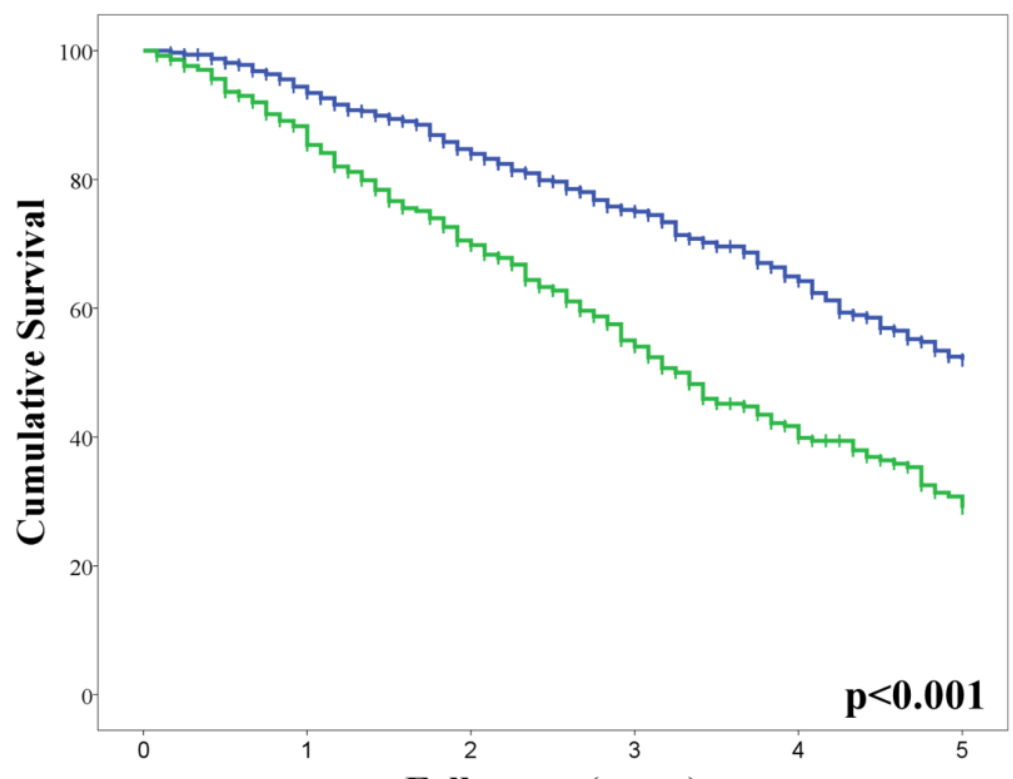

LS (-\%)

$<-10 \%$

$\geq-10 \%$

Follow-up (years)

Number at risk

$<-10 \%$

639

581

450

284

$\geq-10 \%$

507

425

293

172

$\begin{array}{cc}178 & 106 \\ 91 & 52\end{array}$

Figure 2

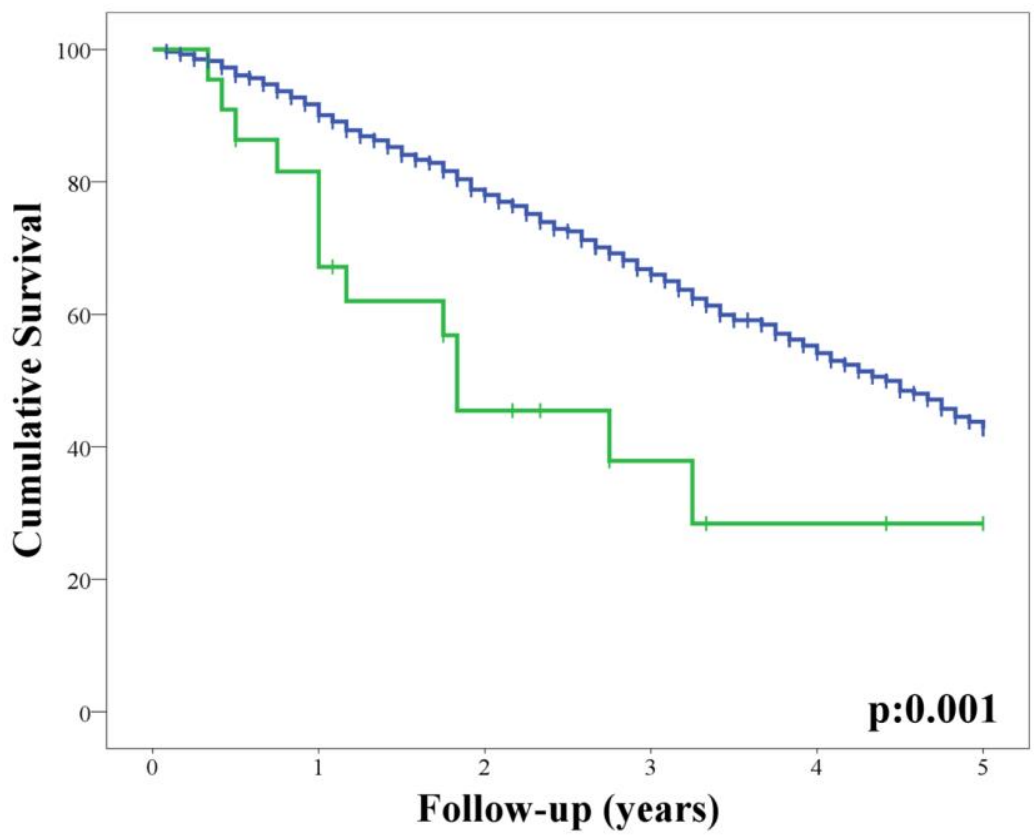

Number at risk

Severe AS NO

$1213 \quad 1060$

783

486

Severe AS

NO

YES

Severe ASYES

22

17

8

4

$\begin{array}{cc}292 & 170 \\ 2 & 1\end{array}$ 
Figure 3

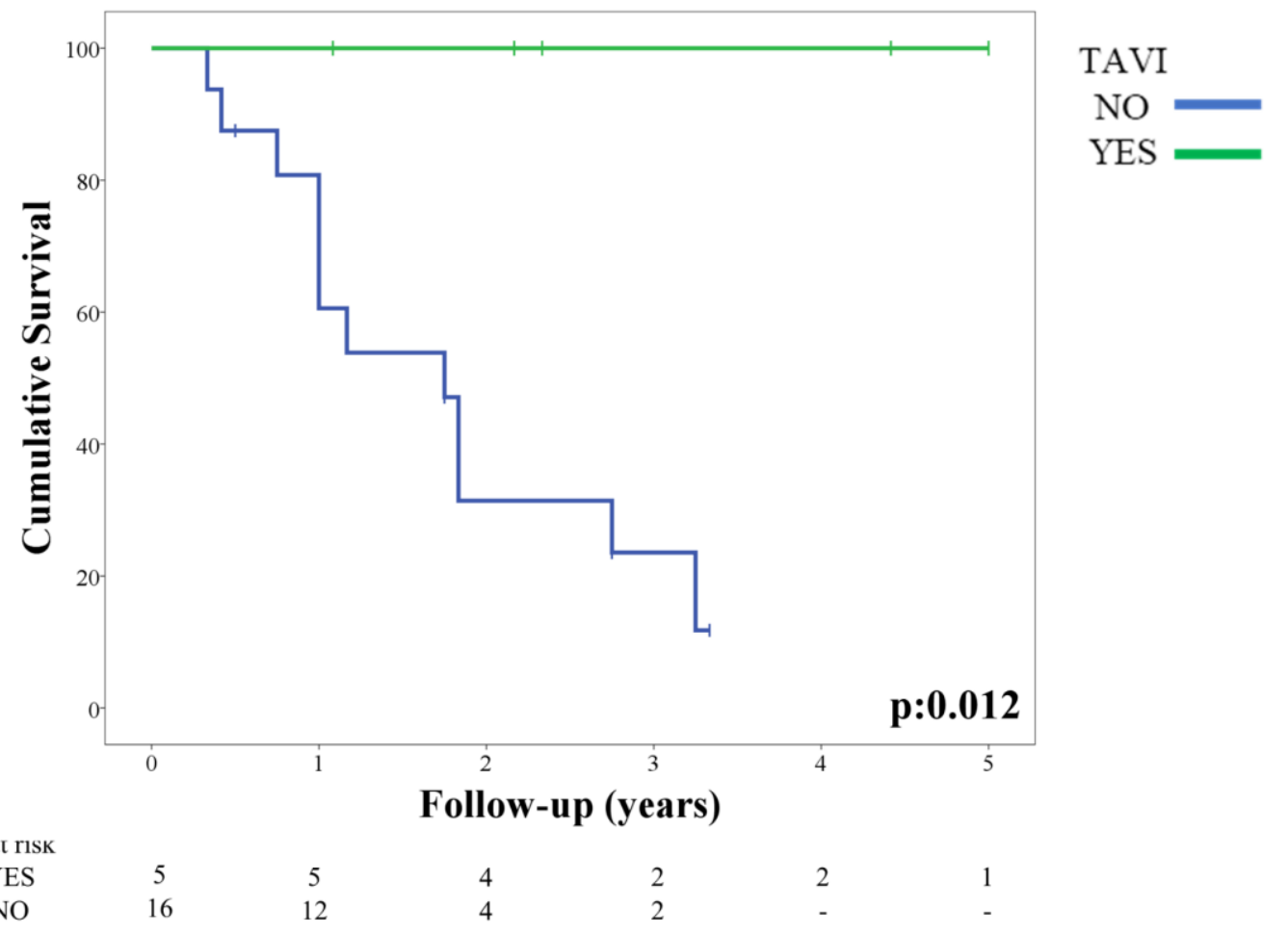

